\title{
Green Cloud Computing: Energy Efficiency and Management
}

\author{
${ }^{1}$ Sumit Kumar*, ${ }^{2}$ Himani Sharma, ${ }^{3}$ Gurpreet Singh \\ ${ }^{1,2,3}$ Department of Computer Science and Engineering \\ ${ }^{1,3}$ CGC College of Engineering, Mohali, Punjab, India \\ ${ }^{2}$ Chandigarh Engineering College, Mohali, Punjab, India \\ Email ID: *sumit.cse@yahoo.com
}

\begin{abstract}
As cloud computing services become popular, it is important for cloud service providers (C SPs) to ensure their duty to society by reducing the impact on the environment due to their operations. C SPs use higher amounts of energy because of the large power demand to run datacenters (DCs). The transient development of cloud computing models has led to the establishment of multiple DCs worldwide. Energy efficiency of IC Tand CO 2 emissions is a major issue in cloud computing. Scientists are constantly working on th is issue to find a solution. Among other options, there is well-known virtualization that is accepted by IT organizations to re duce $\mathrm{CO} 2 \mathrm{emissions}$ and power usage. The basic goal of this paper is to present important methods of VM placement u sed for measurement of PUE in distributed DC s and with varying carbon emission rates. Finally, we present an analysis of several open stack techniques with ACO, DVFS, ECE, and Two-phase Carbon Aware techniques.
\end{abstract}

Keywords: Data center (DC), VM placement, Green IT, Carbon emission, Power Usage Effectiveness (PUE), Carbon Usage Effectiveness (CUE).

\section{INTRODUCTION}

Cloud computing is growing fast by advancing management in the use of computing resources. CSPs provide resources through virtualization where users can use it anywhere and anytime. Among many, the three main CSPs are Microsoft Azure, Amazon Web Services and Google Cloud Platform[1]. Cloud computing offers different administrations for different reason .Its primary three adminis trations: Infrastructure as an administration (IAAS), programming as an administration (SAAS), and stage as an administration (PAAS). Foundation as an administrator allows the client to run their application in type of VMs on a conveyed framework. The application phase as PAAS is a type of computing that enables the platform for customers to build, manage and run an application without compromising quality, and the framework further associated with SAAS is a type of programming platform. In recent years, datacenters (DCs) are continuously increased in its size and counting [2]. These centers measure considerable dependence on res ources and support for its routine operations. In the next few years DC needed the answer for lesser the value and minimum of the quality of management. It is potential by applying the pre-defined metrics and creating the proper activity it is the potential to higher utilize invariably on DC infrastructure and scale back the continual Information and communications technology (ICT) price. ICT consumes the most quantity of power and also mainly it is absorbed by the DCs. The key element of this totalpower consumed by DCs may be a significant collected in scheme carbon intensity. In keeping with researchers, the ICT business produces carbon $2 \%$ of the worldwide greenhouse emission that places it on par with the aviation business [3]. Thus reduction of tiny fractions of energy consumption is a considerable saving in monetary and carbon emis sion of the scheme. The world ICT infrastructure is calculable the consume fifteen, $00 \mathrm{TWH}$ of electricity, roughly usage $10 \%-15 \%$ [4]. ICT account for two carbon emis sion with the DC is chargeable for $14 \%$ of the overall ICT footprints [5]. The functioning price of DCs square measure principally different value square measure personnel connected. Hosts are chargeable for $45 \%$ of the cost, after that infrastructure (25\%), energy consumption (15\%), and internet(15\%)[6]. The cloud center advantage of virtualization technology to share the physical server resource with multiple VMs and make user gets facility wherever, by any kind of terminal[7].

Every VM has its specifications and characteristics counting on res ourceusage. It abs orbs power and releas es $\mathrm{CO} 2$ emis sion. VM deployment for every new $\mathrm{VM}$ request, the cloud administrator chooses the physical machine (PM) to initiate the request in the cloud network [8].If it is finished the correct method it should result in consume lesser energy usage and carbon footprint and the other way around. The energy potency of the system is outlined because of the quantitative relation of helpful tasks done by the system to the overall energy delivered to it. Understanding and analyzing DC metrics allows the operator to read more on potential inefficiencies by focusing on key parameters. Within the DC, the energy potency develops into the helpfulwork performed by alternative systems the foremost widespread energy potency metric-PUE is employed by most of the opposite metrics in direct or indirect kind. The most content of this paper is a comparativestudy of the various techniques that square measure accustomed to maintain energy in the DC and conjointly maintained greenhouse emission in setting on VMs and PMs, while working or replacement. The arrangement of VMs to PMs and the other way around is finished by utilizing different methods. There are an excessive number of systems which are utilizing in conveyed server farms for condition maintainability.

ICT is observing the level of CO2 discharge and PUE range to keep the earth from expanding the rate of carbon impressions. So we should moveto a Green cloud for ecoaccommodating. The developing requests of clients for 
processing administrations are empowering the cloud specialist co-ops e.g., Google, Microsoft, Yahoo!, and so forth to send expanding measures of vitality hungry server farms. Energy is required for support, screens, and system peripherals, cooling fanatics of processors, light and cooling framework and thus, the vitality utilization of the data industry is expanding. Also the data industry is migrating from database to cloud database[9]. The aggregate power utilization of the servers in 2012 was approximately 53GW and about 53\% extraenergy utilizationas compared with2015. This aggregate energyhas been sufficient for satisfying the vitality necessities of every single private family unit of the United Kingdom.

\section{LITERATURE REVIEW}

Many of the investigators have done their analysis in this field however several areas still need more analysis regarding a way to scale back the consumption of energy and minimize the carbon footprint. A carbon footprint described as a complete measurement of greenhouse gas emission also expressed as tons of carbon dioxide (CO2) emission. The most important one from every issue discussed here is introduced i.e. cost. There are unit immeasurable techniques used to minimize the energy effectiveness VMs.

Atefeh Khosravi et al [10] introduced an algorithm for Energy and Carbon-Efficient (ECE) VM placement algorithm that may be a source of derivative to best fit to put the Virtual Machines (VMs) within the data centre, grid, and server with the least amount of CO2, PUE, and limited increment in servers' energy usage. They additionally think about reducing the carbon footprint of Cloud DC sites.

Aksanli, Goir et al[11]further analyzed ECE VM placement method works is finished by VM placement algorithmic program for distributed cloud data centres. Experiment output proves planed algorithmic program decreases the dioxide discharge and energy utilization, whereas it keeps an equivalent stage of QOSin analogy with alternative emulative algorithms. The PUE worth relies on work by linguist et al. and it varies [1.56-2.1] and different CO2 emission rates [0.730]. The registration of every DC website with its characteristics is to be drained ECE cloud info service system (ECE-CIS) and therefore the ECE-CIS update their info time by time.

A. Verma et al[12] emphasis on main propose of Energy and Carbon-Efficient (ECE) VM placement algorithm is to optimize the energy potency in DCs with totally dissimilar CO2 emission rates and Power Usage Effectiveness. The knowledge is embodied in their physical resources, energy parameters like PUE worth, carbon footprint rate etc. Cloud service supplier is employed with this info in the cloud computing atmosphere to perform VM placement with ECE.To evaluate the rigorous performances with this approach CLOUDSIM toolkit is to be used. Through an experiment, it offers the numerous gains as in interval and value saving below dynamic employment atmosphere.

Nathuji R, Schwan K[13]introduced a new technique to minimize the carbon rate and resource called "Distributed virtualization frequency scaling (DVFS)" technique. This technique comes under the "Dynamic System Performance Scaling (DSPS)". This technique adjusts theperformance of the component according to the resource's demand and system states (current and previous). Also this technique is us eful for system parts to maintain the dynamic change of its functionality proportionally with power consumption. It is fully deactivated approach when the system is in idle state (CPU, memory and other) and some component of the system supports in increase and decrease the clock frequency along with the adjustment of the supply voltage at the time of system's idle state. This is a hardware-level technique of dynamic power consumption which is based on clock frequency.

T. Mastelicet al[14] discussed the limitation of this method is that it can be used only in homogeneous systems and not in heterogeneous systems. Another limitation of this technique is, that a control law cannot be designed to satisfy any given performance objective, as long as control efforts are finite. They are important in engineering applications, where resources are always limited. Fundamental performance limitations indicate that there is a need to optimize the control under the given constraints, change the network design, or revert to alternative control strategies for which the limitations do not apply. Reduction in temporary is system. This technique is not successful in large network implementation. The switching circuit becomes complicated as per requirements is increased. The coherence problem is there is a distributed network.

F. Kong and X.Liu[15]reviews the green energy-aware scheduling based algorithm. Under this author introduced a new technique named "Two-Phase Carbon Aware Cloud Broker" has been proposed to try to reduce power and carbon by analyzing the energy and carbon performance of DCs. The suggested method assumes three central entities: user, cloud server, and green cloud broker. Firstly, Users call to perform their cloud jobs (Cloudlet) to the broker. Each cloudlet has a length in terms of the number of instructions having a Million instructions (MI) as its unit. Further, the Cloud server provides services on rent, payments as use applications.

C. Blum et al.[16] projected the estimating power consumption and carbon footprints methods to grade the providers and their DCs. The framed assumed that all requested are coming from users are inhomogeneous environments but this is not happened in the real world, having the heterogeneous request and distributing in the way to optimize the resources. Under infrastructure as a service (IAAS), infrastructure is on lease. DCs are known as a set of physical machines (PMs) with their resources. So every cloud service provider is liable for relevant parameters like PUE, CO2 emission value, and PMs. Moreover, Green Cloud Broker (GCB) duty is almost the equivalent as general Cloud Broker(CB), having exception that GCB being able to compute the $\mathrm{CO} 2$ emission rate of the cloudlets.

Amritpal Kaur et al[17]suggested a method to reduce greenhouse gas emission and energy loss in the DC, regarding the green part of the DC and various powersaving methods have also been surveyed for efficient use of resources. 
R. Brown et al[18]conducted a thorough review of an infrastructure maintaining the cloud computing model about power efficiency. The author aims to focus on the energy efficiency of ICT devices such as hosts and structures, and software services operating over ICT devices such as cloud management systems (CMS) and devices.

D. Kumar et al[19]proposed a genetic algorithm structure that has been recommended for job scheduling to reduce energy consumption in cloud infrastructure. The cloud services broker approach adds quality and also works on its pros and cons. The broker may be a layer that lies between the cloud service and supplier also as an organization. If we tend to do a discussion on security then it is not secured. Reliance is one of the telecom companies as a cloud broker, regularly working with its small or medium-sized team with options for new technologies and offers.

Marco Dorigo et al[20]projected another improvement technique called the Ant Colony Optimization (ACO) algorithm that can be a probabilistic technique for the decision process. The most important objective of the algorithm is the VM placement solution (looking for the shortest and simplest route), which can be used by the physical server to return the total resource wastage (central processor, memory, optional storage devices) and power consumption. It provides the power of fault tolerance by load equalization. Solution via this system tells us that VM must be placed on that server. The researchers conferred a framework to manage the VM placement using an algorithm for the IAAS setting.

$S$. Wibowo et al.[21]outlined an initial VM placement strategy and projected multi-objective improvement ACO to work out the initial VMs placement. Additionally, ACO invoked the

Virtual machine[22]placement drawback thanks to its polynomialtime worst-case quality and convenience of parallelization [23]. This algorithmic rule is predicated on ACO to realize each measurability and high DC utilization by applying VM consolidation[24]. All solutions are hierarchal in line with their length. The quantity of secretion deposited is then weighted for every answer, specified solutions with shorter ways deposit a lot of secretion than the solutions with longer ways. this system is not flourishing in programming algorithms (larger) like Single machine total weighted timing drawback(SMTWTP), Resource based constrained project programming drawback (RCPSP)[25], [26].

Richard Yoo et al[27]proposed a new technique called "Open Stack (OS)", is one of the generally accepted private cloud computing tools.It provides a robust cloud computing resolution with elements namely Neutrons, Heat, and Nova.The Open Stack launched in 2010 is a joint scheme between Rack-space with NASA. From 2016, it is run by the Non-profit Open Stack Foundation. This further presents a tool for DC control described as live migration, which allows virtual machines shifted to a separate server without its turning off and control host live status.The Open Stackplan aims to offer companies cloud solutions operating on standard tools. This feature of Open Stack can be further control and make use to provide an automatic DC control service. This analysis is based on the outcomes and review of previous studies conducted on active VM deployments and has been expanded to be an intelligent DC management tool to make smart decisions. There is a focus on internal parameters of the PM as well as the external parameters within the DC to make smart decisions by utilizing Open Stack live migration[28]. Its computing structure is planned for horizontal scaling on common hardware and software specifications and provides the ability to combine with the right systems and third-parties.

\section{REVIEW AND ANALYSIS}

Data centre uses modern cooling techniques are developing and attracting the attention of administrators, particularly in the big DC operation[29].There are alot of methods that are used in quality improvement, decrease vitality utilization and carbon impression. Sever always area units effectively operating and a few of them have the most extremely dangerous outcomes. TABLE I. COMPARISON OF DIFFERENT TECHNIQUES
BASED UPON PUE, CUE AND COST

\begin{tabular}{|c|c|c|c|c|c|c|c|}
\hline $\begin{array}{l}\text { Tech. } \\
\text { Name }\end{array}$ & PUE & $\begin{array}{l}\text { CUE }= \\
c f^{*} \text { PUE }\end{array}$ & $\begin{array}{c}\text { Cost }= \\
T E(d c)^{*} \text { Price }\end{array}$ & $\begin{array}{l}\text { Dat } \\
\text { Heter } \\
\text { Hom }\end{array}$ & $\begin{array}{l}\text { center } \\
\text { ete: } \\
\text { geneous } \\
\text { mo: } \\
\text { genous }\end{array}$ & Result & $\begin{array}{c}\text { Load }=\text { No. of } \\
\text { (data center }(i=1)^{*} \text { cluster }(j=1) \\
\left.{ }^{*} \text { Host }(k=1)^{*} t\right) v m(i, i, k)\end{array}$ \\
\hline $\begin{array}{l}\text { Energy \& } \\
\text { carbon } \\
\text { efficient }\end{array}$ & $\begin{array}{l}1.56 \\
-2.1\end{array}$ & $\begin{array}{l}0.124- \\
0.730\end{array}$ & Reduce & Hete & Homo & $\begin{array}{l}8 \%-<20 \% \\
\text { Power } \\
\text { Consumpti } \\
\text { on }\end{array}$ & $\begin{array}{l}\text { Min30-max(Single to multiple } \\
\text { data centers) }\end{array}$ \\
\hline DVSF & $>1$ & Lesser & $\begin{array}{l}\text { Acc. to } \\
\text { resource } \\
\text { usage }\end{array}$ & Hete & Homo & $\begin{array}{l}\text { Increase } \\
\text { efficiency } \\
\text { by } \\
\text { reducing } \\
\text { idlewaste } \\
\text { \&scale } \\
\text { down of } \\
\text { wimpy } \\
\text { CPUs }\end{array}$ & $\begin{array}{l}\text { Max(Thousands\& Single- } \\
\text { Multiple data centers) }\end{array}$ \\
\hline$\overline{A C O}$ & $>=1$ & Lesser & $\begin{array}{l}\text { Resource } \\
\text { usage }\end{array}$ & - & Homo & $\begin{array}{l}\text { Reduce } \\
\text { Power\& } \\
\text { resulting } \\
\text { from } \\
\text { resource } \\
\text { wastage }\end{array}$ & $\begin{array}{l}\text { Single-Multi \& (Single multi data } \\
\text { centers) }\end{array}$ \\
\hline $\begin{array}{l}\text { Open } \\
\text { Stack }\end{array}$ & 1.2 .2 & $0.05-0.10$ & Open Source & Hete & Homo & $\begin{array}{l}\text { Prevent } \\
\text { from } \\
\text { disasters }\end{array}$ & $\begin{array}{l}\text { Max(Thousands)(Single- } \\
\text { Multiple data centers) }\end{array}$ \\
\hline $\begin{array}{l}\text { Two } \\
\text { Phase } \\
\text { carbon- } \\
\text { aware } \\
\text { broke }\end{array}$ & $\begin{array}{l}1.56- \\
2.1\end{array}$ & $\begin{array}{l}0.124- \\
0.730\end{array}$ & Effective & Hete & Homo & & \\
\hline
\end{tabular}

- ECE saves 100 percent and 45\% carbon footprints in system.it scale back power consumption in DC up to $8-<20 \%$ and it scale back overall running price of knowledge centres as a result of it will run on completely different core(process ors).

- DVFS technique will give an economical direction in managing power consumption of the hardware. This technique deactivates the PM once it's in idle state and thereon once there ought to meet the demand.

- ACO technique met heuristic with balanced resource utilization. This pseudo based mostly algorithmic rule scale back the energy price and therefore the time for looking possible resolution.ACO invoked the Virtual Machine deployment downside to its polynomial-time worst-case quality and support.

- Open stack technique price-effective and it prevents from disaster to DCs. 
- Two-phase carbon aware scale back carbon emission by 2 hundredth and maximize the energy and conjointly introduce the conception of in experienced carbon. During this user will work on countless instructions (MI).

Throughout this paper we tend tothought-about and examined different systems that depend upon few parameters (predefined -regulate) to prolong many actualities that show that strategy is healthier and not constant as an alternative one that of the conditions causes specialists to complete their investigation.

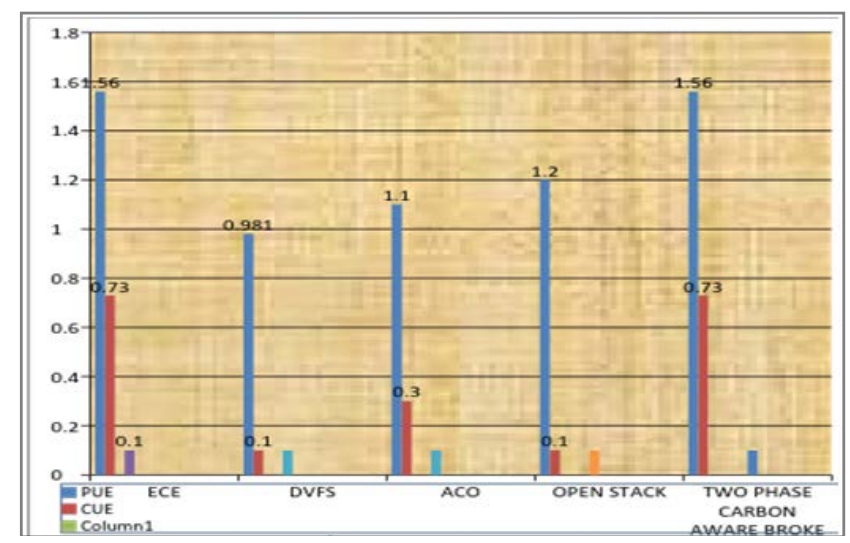

Fig. 1. Comparison graph of PUE and CUE with respect ofdifferent techniques

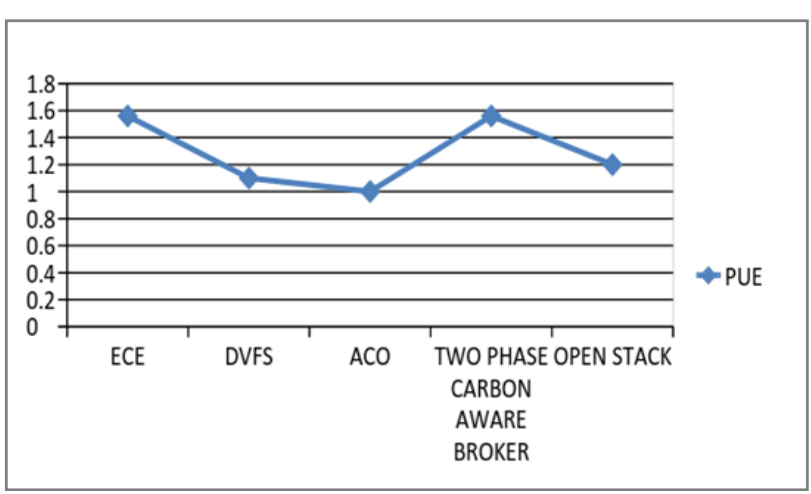

Fig. 2. Comparison graph of different techniques based on Power Usage Effectiveness (PUE)

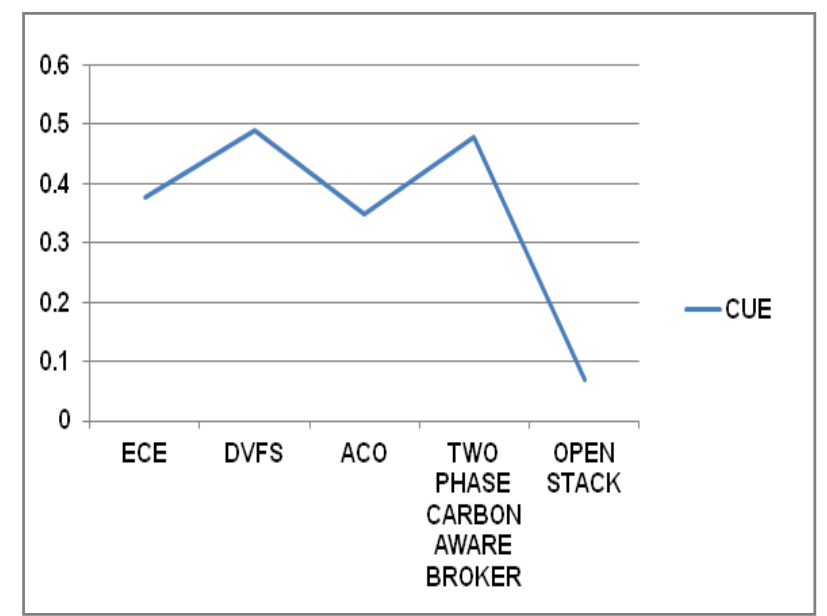

Fig. 3. Comparison graph of different techniques based on Carbon Usage Effectiveness (CUE)
TABLE II. DIFFERENCE BETWEEN TECHNIQUES BASED UPON PUE

\begin{tabular}{|l|c|}
\hline \multicolumn{1}{|c|}{ Technique name } & PUE \\
\hline ECE & 1.56 \\
\hline DVFS & 1.1 \\
\hline ACO & 1.0 \\
\hline $\begin{array}{l}\text { TWO-PHASE CARBON AWARE } \\
\text { BROKER }\end{array}$ & 1.56 \\
\hline OPEN STACK & 1.2 \\
\hline
\end{tabular}

TABLE III. DIFFERENCE BETWEEN TECHNIQUES BASED UPON CUE

\begin{tabular}{|l|c|}
\hline \multicolumn{1}{|c|}{ Technique name } & CUE \\
\hline ECE & .378 \\
\hline DVFS & 0.49 \\
\hline ACO & 0.35 \\
\hline $\begin{array}{l}\text { TWO-PHASE CARBON AWARE } \\
\text { BROKER }\end{array}$ & 0.478 \\
\hline OPEN STACK & 0.07 \\
\hline
\end{tabular}

\section{RESUlts AND Discussions}

In this paper we have mentioned about cloud computing with its numerous virtualization techniques that are extremely in style in present days. Utility computing becomes support for cloud and grid computing, which uses the concept of virtualization[30].We has got mentioned the techniques of VM placement to cut back the energy consumption and carbon footprint. Techniques may work success fully only on their compatible environment. As shown in Table1 several techniques provide sensible leadsto consiste nt surroundings and a few of them in uniform DC. Wet end to earlier discuss on DVFS technique, this method works on each heterogeneous and consistent DC however this method offers higher ends up inconsistent settings instead of heterogeneous by experimentation. This is often shifting and hardware-based mostly dynamic power consumption technique within which reduction is additionally temporary whereas implementation. Ant colony optimization (ACO) is employed to model the core infrastructure-level hardware services that are obtainable by cloud suppliers. It encapsulates a collection of cipher hosts which will either be consistent or heterogeneous.

The disadvantage of ACO is its heuristics construction that offers solely restricted variety of solution or is generated. To boot, greedy choices in early stages of the development method powerfully constrain on the on the market potentialities at later stages, typically determinative poor moves within the final phases of the answer construction. This method is simply for brief comes. Its value is predicated on resources. The worth of PUE $(>=1)$ and carbon footprints rate $(<1)$ in the ACO and DVFS technique. As shown inFig.3. ECE provides higher results as compared to ACO and DVFS techniques; it uses the CLOUDSIM toolkit which provides smart results choose interval and price with ' $n$ ' complexness reduction. The two-section carbon technique is additionally a decent one technique. This 
method is additionally accustomed to cut backcarbon emission. The modal of this method is constructed below advanced calculations. It needs double of resources for workn complexness of this method is on top of different. There is great pressure on memory and storage devices with networking between them. This is often affordably needed special necessities. The open stack technique is recently launched for distributed centres. In case of distributed computing, it provides secure access to a client for ICT management[31].It may work on each heterogeneous and consistent centre with less carbonic acid gas emission and energy potency rate. It is a type of open supply that makes it less expensive than relatively different technologies. There's no special demand for hardware and package, with this feature it optimized the resource allocations or requirements and also effective to reduce power consumption and carbon footprints rate. This technique prevents disasters. This feature is made it unique fromothers. It overcomes all of the limitations which we have to face other technique with other techniques, experimentally.

As stated in the McKinsey report[32], "The total estimated energy bill for DCs in 2010 is 11.5 billion and energy costs in a typical DC double every five years". In reality the energy utilization is expected to increase by $76 \%$ from 2007 to 2030 [33]. Also, the environment is threatened by large amounts of CO2 emissions from IT companies, so the demand to achieve more environmentally friendly green computing increased. This leads to efforts to maximize energy efficiency and utilization to reduce costs and carbon emission rates. The foremost goal of Green Computing is to find novel machines, computing prototypes, and services with minimal cost and energy use and development of society. [15]. Based upon the value of PUE and CUE as shown in Fig. 2 and Fig. 3 is evaluated on the bases of resources allocation (IT equipment) which were used in VM placement about different techniques. The proposed methods about the efficient assignment of tasks based on green computing in a private cloud[34]. The suggested procedures include energy-saving in a green cloud, which us es clustering at the VM level to classify the machine as low- and high-level VMs. Cloudlets are also classified into three sections: Suspend able, Cancellable, and Non-per mutable cloudlets. They studied the energy consumption of complex workloads as different processing components, such as the number of CPUs needed by Cloudlet to improve the performance of compression methods. Cloud applications are also used in the field of VANET which are usually equipped with sensors that process accurate information to route data packets, which manage data and transmission delays from redundancy with the help of cloud technology[35]. The above-discussed techniques provide a way to move towards Green Cloud Computing. However there are chances to improve the values of PUE \& CUE by including Non-IT equipments.

\section{CONCLusion AND Future SCOPE}

In the future few years, the migration of VMs is going to be feasible with less utilization of resources and power consumption. Numbers of approaches suggested scaling back carbon emission and energy consumption up to $15 \%$ -
$20 \%$ as compared to others. We conclude that this review can be a sensible recommendation for investigators in coming up with algorithms that perform customer tasks in DCs. The main purpose of the study is the effect of various user utility and VM deployment strategies. During this paper we conduct a comparative study of many techniques. Open stack technology provides better results than ACO, DVFS, ECE, and Two-Phase Carbon Aware Broker techniques, due to its options. In the future, there is a need to explore the results of $\mathrm{CO} 2$ emissions, inter-datacentre network range, and information section on the cloudautomated data processing method.

\section{REFERENCES}

[1] H. S. Sumit Kumar*, Sunil Kumar, "A Comparitive Study of various Cloud Service Platforms,” 2019.

[2] Corey Gough, I. Steiner, and W. Saunders, Energy Efficient Servers: Blueprints for Data Center Optimization. Apress, Berkeley, CA, 2015.

[3] M. P. Mills, The cloud begins with coal - An overview of the electricity used by the global digital ecosystem. 2013.

[4] A. Greenberg, J. Hamilton, D. A. Maltz, and P. Patel, “The cost of a cloud," ACM SIGCOMM Comput. Commun. Rev., vol. 39, no. 1, p. 68, 2008.

[5] “AWS T otal Cost of Ownership (TCO) Calculator.” [Online]. Available: https://awstcocalculator.com/. [Accessed: 17Apr-2019].

[6] "VMware TCO Comparison Calculator." [Online]. Available: VMware TCO Comparison Calculator. [Accessed: 17-Apr2019].

[7] A. Rashid and A. Chaturvedi, "Cloud Computing Characteristics and Services A Brief Review," Int. J. Comput. Sci. Eng., vol. 7, no. 2, pp. 421-426, 2019.

[8] F. Ma, F. Liu, and Z. Liu, "Multi-objective Optimization for Initial Virtual Machine Placement in Cloud Data Center $\star, "$ J. Inf. Comput. Sci., vol. 9, pp. 5029-5038, 2012.

[9] G. Singh, S. Kumar, S. Chawla, and A. Professor, "Moving from databases to Cloud Database: Futuristic Trends," CGCIJCTR, vol. 1, no. 2, pp. 12-16, 2019.

[10] A. Khosravi, S. K. Garg, and R. Buyya, “Energy and carbonefficient placement of virtual machines in distributed cloud data centers,” Lect. Notes Comput. Sci. (including Subser. Lect. Notes Artif. Intell. Lect

[12] A. Verma, R. Koller, L. Useche, and R. Rangaswami, "SRCMap: Energy Proportional Storage using Dynamic Consolidation,” Energy, no. VM, p. 20, 2010.

[13] R. Nathuji and K. Schwan, "VirtualPower: Coordinated Power Management in Virtualized Enterprise Systems," ACM SIGOPS Oper. Syst. Rev., vol. 41, no. 6, p. 265, 2007.

[14] T. Mastelic, A. Oleksiak, H. Claussen, I. Brandic, J.-M. Pierson, and A. V Vasilakos, "Cloud Computing: A Survey of Energy Efficiency,” ACM Comput. Surv., vol. 47, no. 2, pp. 1-36, 2014.

[15] F. Kong and X. Liu, "A Survey on Green-Energy-Aware Power Management for Datacenters,” ACM Comput. Surv., vol. 47, no. 2, pp. 1-38, 2014.

[16] C. Blum, "ACO Applied to Group Shop Scheduling: A Case Study on Intensification and Diversification,” pp. 14-27, 2007.

[17] A. Kaur and S. Kinger, "Increasing Cloud Usage: A Shift towards Green Clouds,” Int. J. Comput. Appl., vol. 67, no. 13, pp. 28-32, 2013. 
[18] R. Brown, A. to S. Energy, I. Incorporated, and E. Al., Report to Congress on Server and Data Center Energy Efficiency: Public Law 109-431, no. 2008-06-13. 2008.

[19] D. Kumar, B. Sahoo, B. Mondal, and T. Mandal, “A Genetic Algorithmic Approach for Energy Efficient Task Consolidation in Cloud Computing,” Int. J. Comput. Appl., vol. 118, no. 2, pp. 1-6, 2015.

[20] A. Colorni, M. Dorigo, and V. Maniezzo, “An investigation of some properties of an 'Ant algorithm,'” Ppsn 92, no. Ppsn 92, pp. 509-520, 1992.

[21] P. N. B. L. N, S. Wibowo, and M. Wells, "Green Cloud Computing and Economics of the Cloud: Moving towards Sustainable Future,” vol. 5, no. 1, pp. 15-20, 2016.

[22] “Amazon EC2 Instance Types.” [Online]. Available: http://aws.amazon.com/ec2/instance-types.

[23] F. D. Methods, " 2 The Single Machine Total Weighted Tardiness Problem 3 ACO Algorithm for SMTTP," Evaluation, pp. 287-296, 2000.

[24] R. Talaber, V. M. Ware, and L. Lamers, "Using Virtualization To Improve Data,” pp. 1-22, 2009.

[25] M. Den Besten and T. Stutzle, “An Ant Colony Optimization Application to the Single Machine Total Weighted Tardiness Problem,” no. February 2014, pp. 14451450, 2000.

[26] M. S. Tiwari V, Ashar P, “Technology mapping for low power," in 30th ACM/IEEE Design Automation Conference, 1993.

[27] R. Nathuji, C. Isci, and E. Gorbatov, "Exploiting platform heterogeneity for power efficient data centers," Fourth Int. Conf. Auton. Comput. ICAC’07, 2007.

[28] "Comparing OpenStack and AWS Total Cost of Ownership.” [Online]. Available: https://www.mirantis.co.jp/comparing-openstack-and-awstotal-cost-of-ownership/. [Accessed: 17-Apr-2019].

[29] O. Gadgil and K. A. Rahn, "Assessment of Emerging Cooling Technologies by Analyzing Their Impact on Reducing the Power Usage Effectiveness Ratio of Data Centers,” pp. 640-645, 2019.

[30] D. Sood, H. Kour, and S. Kumar, "Survey of Computing Technologies: Distributed, Utility, Cluster, Grid and Cloud Computing,” J. Netw. Commun. Emerg. Technol. www.jncet.org, vol. 6, no. 5, 2016.

[31] D. Kaur, A. Professor, M. Talwar, and S. Kumar, "Controlled Security in Cloud Computing based on RBAC: A Review Analysis," J. Netw. Commun. Emerg. Technol. www.jncet.org, vol. 6, no. 5, 2016.

[32] P. Singh, A. Kaur, M. S. Devgan, and H. K. Toor, "Performance Optimization of IOT Networks Using Frequency Hopping,” CGC Int. J. Contemp. Technol. Res., vol. 0486, no. 2, pp. 41-44, 2019.

[33] M. Battaglieri et al., "US Cosmic Visions: New Ideas in Dark Matter 2017: Community Report,” Jul. 2017.

[34] B. Aksanli, J. Venkatesh, L. Zhang, and T. Rosing, "Utilizing green energy prediction to schedule mixed batch and service jobs in data centers," ACM SIGOPS Oper. Syst. Rev., vol. 45, no. 3, p. 53, 2012.

[35] "Efficient Task Allocation Based on Green Computing in Private Cloud,” vol. 0486, pp. 68-76.

[36] D. Verma and P. Singh, "Safety Message Dissemination in Cloud-VANET based Infrastructure through Game Theory," vol. 0486, pp. 57-61. 